\title{
The period and amplitude variations of $\delta$ Scuti star HD 93044
}

\author{
Liu Zong-Li \\ Beijing Astronomical Observatory, Chinese Academy of Sciences, 100080 Beijing, China \\ INTERNET: lzl@bao01.bao.ac.cn
}

Received February 9, 1994; accepted October 17, 1995

\begin{abstract}
The $\delta$ Scuti variable star HD 93044 was observed with the $60 \mathrm{~cm}$ telescope at the Xinglong Station of the Beijing Astronomical Observatory from 1992 to 1993. Period analyses were made for the 1992 data set, the 1993 data set, and the combined 1992-1993 data set. Besides the known three frequencies: 11.90809, 16.798 and $22.074 \mathrm{~cd}^{-1}$, two new frequencies of 2.086 and $10.489 \mathrm{~cd}^{-1}$ were found. Some amplitude variations of two pulsation components $\left(11.90809,22.074 \mathrm{~cd}^{-1}\right)$ were obvious. The new frequency of $2.086 \mathrm{~cd}^{-1}$ might be the interaction mode from which the resonances can occur and then lead to the nonlinear phenomena in the $\delta$ Scuti variable star.
\end{abstract}

Key words: stars: $\delta$ Scuti — stars: oscillations — stars: individual: HD 93044

\section{Introduction}

HD 93044 is a 7.0 magnitude star situated at $\alpha=10^{\mathrm{h}} 42^{\mathrm{m}} \cdot 5, \delta=+41^{\circ} 34^{\prime} \cdot 3$ (1950 coordinates). Heynderickx (1990) observed this star from 1984 to 1987 at the Hochalpine Forschungsstation Jungfraujoch in Switzerland and obtained one frequency of 11.90808 cycles per day $\left(\mathrm{cd}^{-1}\right)$. Liu Zong-Li (1993) observed this star in the spring of 1991 at the Xinglong Station of the Beijing Astronomical Observatory. Besides $11.90808 \mathrm{~cd}^{-1}$ he obtained two other frequencies $\left(16.795 \mathrm{~cd}^{-1}, 21.97 \mathrm{~cd}^{-1}\right)$ and found some amplitude variations of two pulsation components. In this paper we wish to present and analyze our data from the Xinglong Station of the Beijing Astronomical Observatory, provide a frequency and amplitude analysis of our data, determine the frequency and amplitude variations, and give a possible interaction mode from which the resonances can occur and then lead to these variations.

\section{Observations}

HD 93044 was observed photoelectrically with the $60 \mathrm{~cm}$ telescope at the Xinglong Station of the Beijing Astronomical Observatory from 7 to 15 February in 1992 and from 31 January to 25 May in 1993. The single-channel integration photometer equipped with an EMI6256B photomultiplier was used in the DC mode with the output digitized with a V/F converter (Shi et al. 1987). The Johnson $V$ filter was used. The star HD 93457 was used as comparison, and HD 93664 as check star. No evidence for any variability of these stars was found. During the observing
Table 1. Observing log of HD 93044 (1992-1993)

\begin{tabular}{ccc}
\hline \hline Date (UT) & Time duration (day) & Point number \\
\hline 1992.02 .07 & 0.2484 & 57 \\
1992.02 .08 & 0.3593 & 92 \\
1992.02 .09 & 0.3865 & 80 \\
1992.02 .11 & 0.3722 & 82 \\
1992.02 .12 & 0.3969 & 89 \\
1992.02 .13 & 0.3912 & 110 \\
1992.02 .15 & 0.4178 & 107 \\
1993.01 .31 & 0.3264 & 93 \\
1993.02 .01 & 0.1359 & 42 \\
1993.02 .02 & 0.3041 & 88 \\
1993.02 .03 & 0.1688 & 57 \\
1993.02 .10 & 0.2094 & 61 \\
1993.02 .11 & 0.1185 & 35 \\
1993.02 .12 & 0.3556 & 95 \\
1993.05 .25 & 0.0877 & 26 \\
\hline \hline
\end{tabular}

run in 1992 the weather was very good. A good data set which consists of 617 data points covering about 62 hours was obtained. The measuring error is about $5.5 \mathrm{mmag}$. 497 data points covering about 42 hours were obtained during the observing run in 1993. The measuring error is about 6.5 mmag. The observing log is given in Table 1 , where date is in universal time (UT), time duration is the length of observational run (in days) during each night, point number is the number of independent observations of HD 93044 during each night. The light curves are shown in Fig. 1, where $\Delta V$ is defined to be the $V$ magnitude 


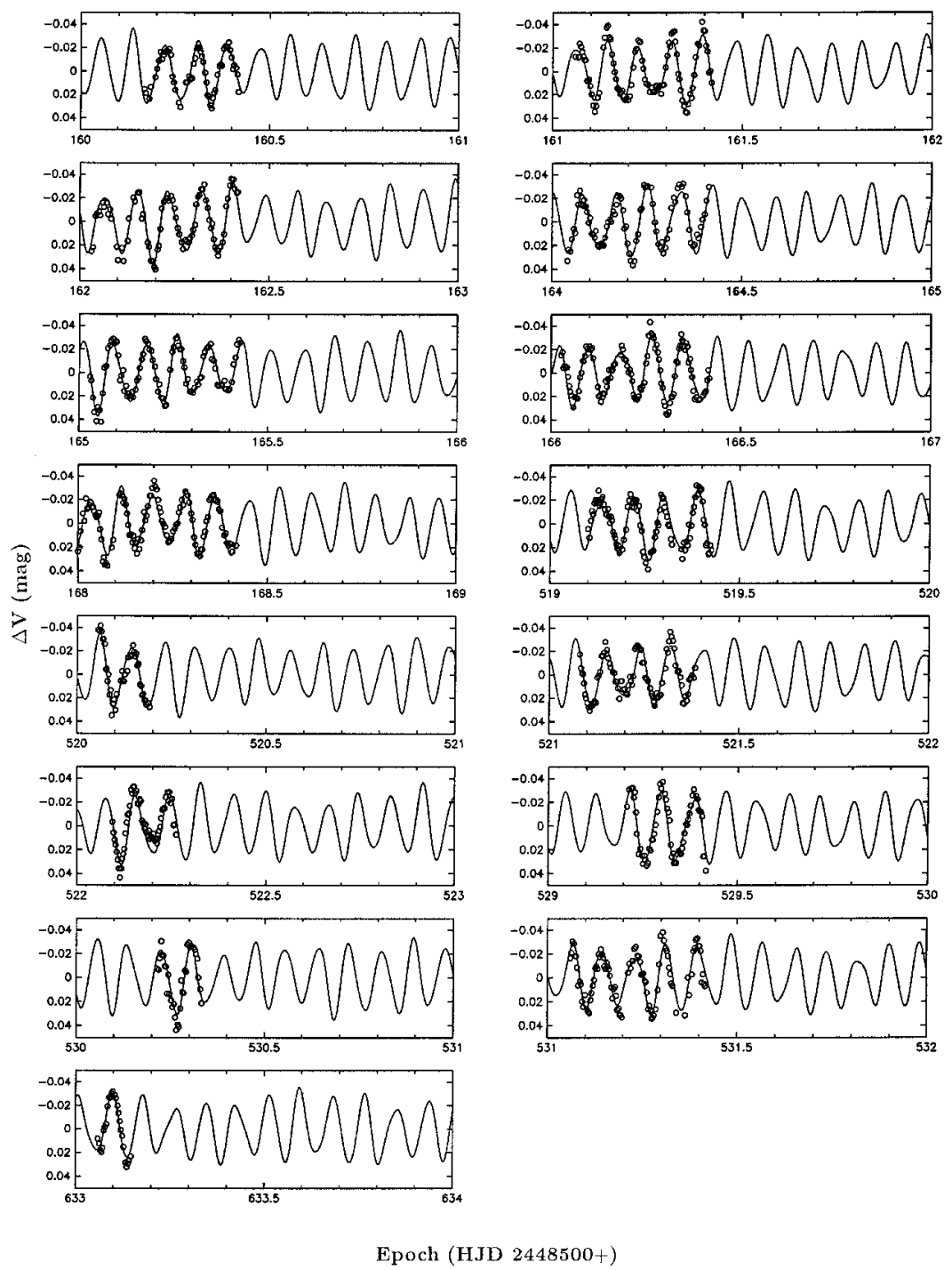

Fig. 1. Light curve of HD 93044 obtained during 1992-1993. $\Delta V$ is defined to be the $V$ magnitude difference (variable comparison star) normalized to zero. The measuring error is about 6 mmag. The fit of the five-frequency solution given in Table 2.3 is shown as a solid curve

difference (variable - comparison star) normalized to zero. The data are available from the author on request.

\section{Period analysis}

The period analyses were performed using the program PERIOD (Breger 1990) and Hao Jin-xin's program (1991). In these programs all parameters of pulsation are obtained using a combination of single-frequency Fourier transforms and multifrequency least squares of brightness residuals (LSR). The function fit to the data is:

$$
m(t)=C+\Sigma_{i} a_{i} \sin \left[2 \pi f_{i}\left(t-t_{1}\right)+2 \pi \varphi_{i}\right],
$$

where $m(t)$ is the predicted magnitude, $C$ is the magnitude offset of the observed data from the fit, $a_{i}$ are the amplitudes (half of the peak-to-peak astronomical amplitudes), $f_{i}$ are the frequencies given in cycles per day, $\varphi_{i}$ are the phases given in units from 0 to 1 , and $t_{1}$ is the time of the first data point.

For each data set the main two steps of the period analysis were as follows:

(1) Doing single-frequency Fourier transforms of the initial data (or corresponding prewhitened data) to obtain the trial frequency. The following step size in frequency was adopted:

$$
\Delta f_{\text {step }}=1 /(20 \Delta t),
$$

where $\Delta t$ is the time interval covered by the observations: $t$ (last) $-t$ (first).

(2) Doing least squares of brightness residuals between trial fit and measurements to find simultaneously the 

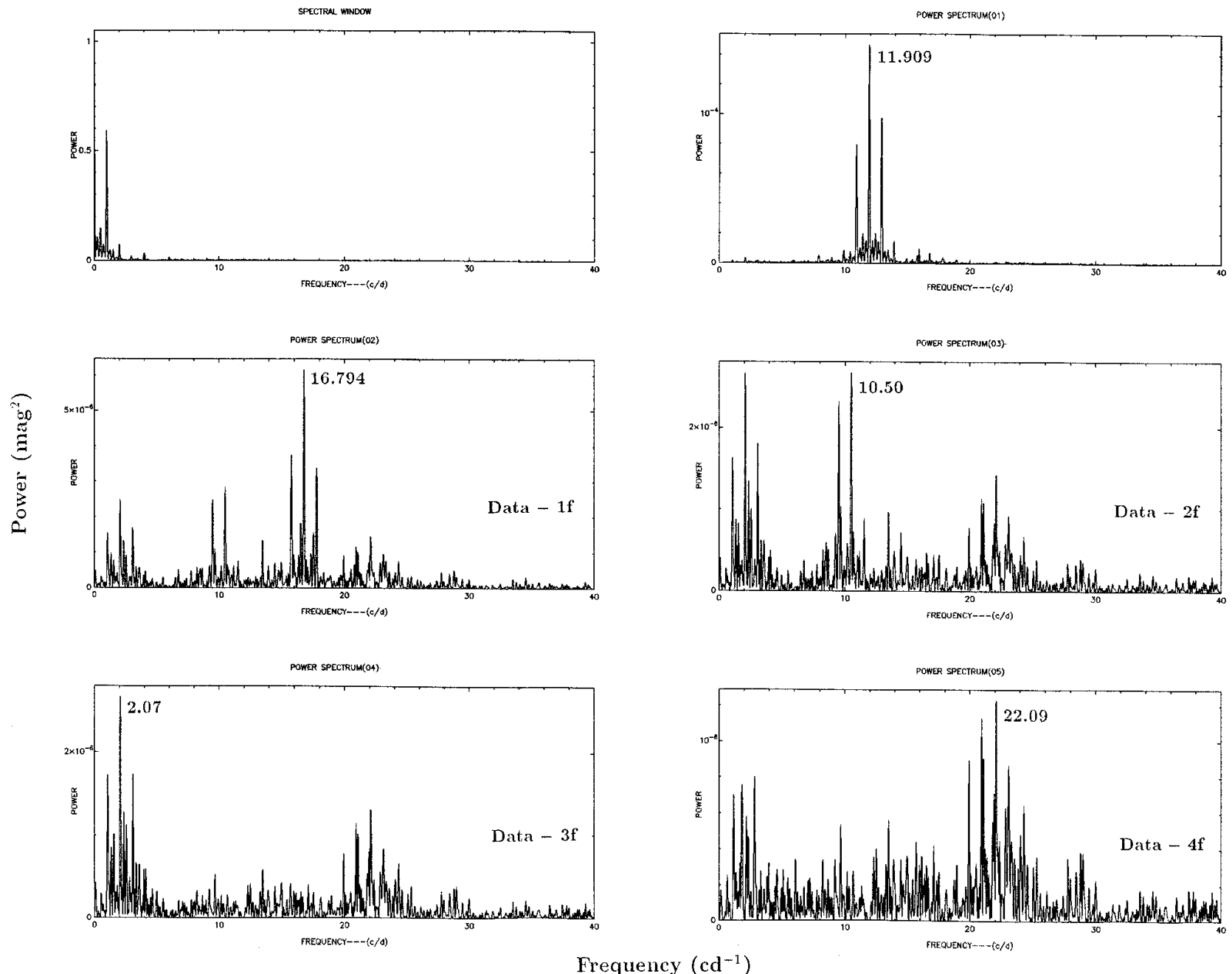

Fig. 2. Power spectra of the 1992 data of HD 93044. Power spectrum (01) is obtained from the original data. The frequency with strongest power is the first frequency of $11.909 \mathrm{~cd}^{-1}$. Power spectrum (02) is obtained from the residuals (data minus first frequency). The frequency with strongest power is the second frequency of $16.794 \mathrm{~cd}^{-1}$. Power spectrum (03) is obtained from the residuals (data minus first and second frequencies). The frequency with strongest power is the third frequency of $10.50 \mathrm{~cd}^{-1}$. Power spectrum (04) is obtained from the residuals (data minus above three frequencies). The frequency with strongest power is the fourth one $\left(2.07 \mathrm{~cd}^{-1}\right)$. Power spectrum (05) is obtained from the residuals (data minus above four frequencies). The frequency with strongest power is the fifth one $\left(22.09 \mathrm{~cd}^{-1}\right)$

frequencies with the smallest residuals between the fit and the measurements.

Repeating the above two steps once a new frequency and related parameters were obtained. Finishing all steps, the final frequencies and related parameters were determined.

Using the above method we analysed the 1992 data first. The computed five-frequency solution is given in Table 2.1. In Table 2 the frequency, amplitude and phase are the parameters of each pulsation mode. The standard error is the fit error of the predicted solution to the data set. The frequency is in cycles per day, the amplitude and standard error are in milli-magnitude, and the value of phase is from 0 to 1 . According to the amplitude of each pulsation frequency the frequency number is given in Col. 3. The frequency with the bigest amplitude is number 1 . The power spectra of the 1992 data are shown in Fig. 2 together with the spectral window. Power spectrum (01) is obtained from the original data. The frequency with strongest power is the first frequency of $11.909 \mathrm{~cd}^{-1}$. Power spectrum (02) is obtained from the residuals (data minus first frequency). The frequency with strongest power is the second frequency of $16.794 \mathrm{~cd}^{-1}$. Power spectrum (03) is obtained from the residuals (data minus first and second frequencies). The frequency with strongest power is the third frequency of $10.50 \mathrm{~cd}^{-1}$. Power spectrum (04) is obtained from the residuals (data minus above three frequencies). The frequency with strongest power is the fourth one 


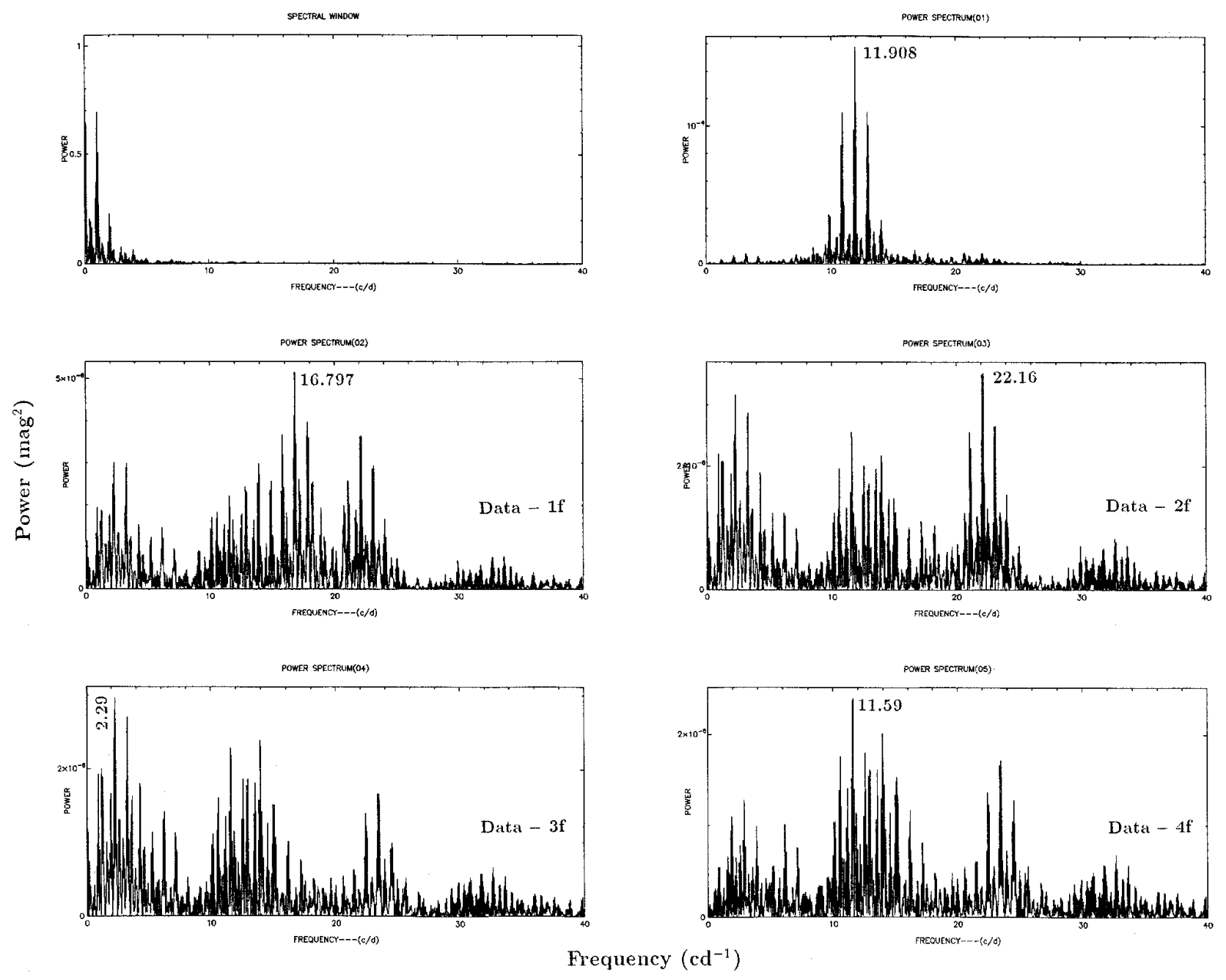

Fig. 3. Power spectra of the 1993 data of HD 93044. Power spectrum (01) is obtained from the original data. The frequency with strongest power is the first frequency of $11.908 \mathrm{~cd}^{-1}$. Power spectrum (02) is obtained from the residuals (data minus first frequency). The frequency with strongest power is the second frequency of $16.797 \mathrm{~cd}^{-1}$. Power spectrum (03) is obtained from the residuals (data minus first and second frequencies). The frequency with strongest power is the third frequency of $22.16 \mathrm{~cd}^{-1}$. Power spectrum (04) is obtained from the residuals (data minus above three frequencies). The frequency with strongest power is the fourth one $\left(2.29 \mathrm{~cd}^{-1}\right)$. Power spectrum (05) is obtained from the residuals (data minus above four frequencies). The frequency with strongest power is the fifth one $\left(11.59 \mathrm{~cd}^{-1}\right)$

$\left(2.07 \mathrm{~cd}^{-1}\right)$. Power spectrum $(05)$ is obtained from the residuals (data minus above four frequencies). The frequency with strongest power is the fifth one $\left(22.09 \mathrm{~cd}^{-1}\right)$. The first frequency $\left(f_{1}\right)$, second frequency $\left(f_{2}\right)$, and the fifth frequency $\left(f_{5}\right)$ are those frequencies which appeared in 1990 and 1991 (Liu Zong-Li 1993). Besides these three frequencies the $10.50 \mathrm{~cd}^{-1}\left(f_{3}\right)$ and $2.07 \mathrm{~cd}^{-1}\left(f_{4}\right)$ are new.

The frequency of $2.07 \mathrm{~cd}^{-1}$ is in the 0 to 3 cycles per day frequency domain which is very important to establish the link between amplitude variability and interactionterm resonances. In view of its importance we observed this star in 1993 again to check its reality. Using the same method, as stated above, we also did the period analysis for the 1993 data. The five-frequency solution is given in Table 2.2. The power spectra of 1993 data are shown in Fig. 3. together with the spectral window. It is obvious that the two new frequencies $\left(f_{4}\right.$ and $\left.f_{5}\right)$ are also present in 1993.

Because the frequency resolution is related to the time interval covered by the observations we did the period analysis for the combined 1992-1993 data using the same method. The five-frequency solution is given in Table 2.3. The values of the five-frequency solution of the 1992-1993 data are more accurate and have more effective figures. The fit of the five-frequency solution given in Table 2.3 to the 1992-1993 data set is shown in Fig. 1 as a solid curve. 
Table 2. Frequency solutions of 3 data sets of HD 93044

\begin{tabular}{|c|c|c|c|c|c|c|}
\hline Heading & $\begin{array}{l}\text { Time } \\
\text { (year) }\end{array}$ & Frequency number & $\begin{array}{c}\text { Frequency } \\
\text { (cycles/day) }\end{array}$ & $\begin{array}{l}\text { Amplitude } \\
\text { (mmag) }\end{array}$ & Phase & $\begin{array}{c}\text { Standard error } \\
(\mathrm{mmag})\end{array}$ \\
\hline \multirow[t]{5}{*}{ Table 2.1} & 1992 & 1 & $11.909 \pm 0.001$ & $24.7 \pm 0.3$ & $0.132 \pm 0.004$ & 5.6 \\
\hline & & 2 & $16.794 \pm 0.004$ & $5.0 \pm 0.3$ & $0.32 \pm 0.02$ & \\
\hline & & 3 & $10.50 \pm 0.01$ & $3.4 \pm 0.3$ & $0.99 \pm 0.03$ & \\
\hline & & 4 & $2.07 \pm 0.01$ & $3.2 \pm 0.3$ & $0.08 \pm 0.03$ & \\
\hline & & 5 & $22.09 \pm 0.01$ & $2.3 \pm 0.3$ & $0.42 \pm 0.04$ & \\
\hline \multirow[t]{5}{*}{ Table 2.2} & 1993 & 1 & $11.9075 \pm 0.0004$ & $24.9 \pm 0.4$ & $0.313 \pm 0.004$ & 6.5 \\
\hline & & 2 & $16.797 \pm 0.001$ & $4.9 \pm 0.4$ & $0.63 \pm 0.02$ & \\
\hline & & 3 & $22.161 \pm 0.001$ & $3.7 \pm 0.4$ & $0.39 \pm 0.02$ & \\
\hline & & 4 & $2.290 \pm 0.002$ & $3.3 \pm 0.4$ & $0.74 \pm 0.02$ & \\
\hline & & 5 & $11.590 \pm 0.003$ & $3.3 \pm 0.4$ & $0.56 \pm 0.03$ & \\
\hline \multirow[t]{5}{*}{ Table 2.3} & 1992-1993 & 1 & $11.90809 \pm 0.00001$ & $24.7 \pm 0.3$ & $0.138 \pm 0.002$ & 6.4 \\
\hline & & 2 & $16.79805 \pm 0.00005$ & $5.0 \pm 0.3$ & $0.30 \pm 0.01$ & \\
\hline & & 3 & $10.4890 \pm 0.0001$ & $3.1 \pm 0.3$ & $0.04 \pm 0.02$ & \\
\hline & & 4 & $22.0740 \pm 0.0001$ & $2.8 \pm 0.3$ & $0.46 \pm 0.02$ & \\
\hline & & 5 & $2.0864 \pm 0.0001$ & $2.8 \pm 0.3$ & $0.01 \pm 0.02$ & \\
\hline
\end{tabular}

\section{Results and discussion}

As stated above, we made period analyses for three data sets. All results obtained are given in two tables and three figures to simplify the discussion. The main results and some discussions are as follows:

1. Heynderickx (1990) observed this star during 19841987 and obtained one frequency. Its value was $11.90808 \pm 0.00002 \mathrm{~cd}^{-1}$. It was $11.90811 \pm 0.00002$ $\mathrm{cd}^{-1}$ during 1990-1991 (Liu Zong-Li 1993). It was $11.90809 \pm 0.00001 \mathrm{~cd}^{-1}$ during 1992-1993 (see Table 2.3). They were exactly the same within the errors. It showed that the first frequency of this star was constant from 1984 to 1993.

2. The $11.909 \mathrm{~cd}^{-1}, 16.794 \mathrm{~cd}^{-1}$ and $22.09 \mathrm{~cd}^{-1}$ in the five-frequency solution of the 1992 data set are old. They were present already during 1990-1991 (Liu Zong-Li 1993). Besides the three frequencies, there are two new frequencies $\left(10.50 \mathrm{~cd}^{-1}\right.$ and $\left.2.07 \mathrm{~cd}^{-1}\right)$ in the five-frequency solution. Their frequency numbers are 3 and 4 among five frequencies (see Table 2.1 and Fig. $2)$. The two new frequencies also appeared in 1993. Their values are $2.29 \mathrm{~cd}^{-1}\left(f_{4}\right)$ and $11.59 \mathrm{~cd}^{-1}\left(f_{5}\right)$, respectively (see Table. 2.2 and Fig. 3). The difference between $10.50 \mathrm{~cd}^{-1}$ (1992) and $11.59 \mathrm{~cd}^{-1}$ (1993) might be due to the aliasing problem. The frequency of $11.59 \mathrm{~cd}^{-1}$ might be one alias of $10.50 \mathrm{~cd}^{-1}$.

3 . The amplitude of the first frequency changed from year to year. It was $24 \pm 1$ mmag during $1984-1987$, then it decreased to $20.9 \pm 0.6$ mmag during 1990-1991 (Liu Zong-Li 1993). And it increased to $24.7 \pm 0.3 \mathrm{mmag}$ during 1992-1993 (see Table 2.3). The amplitude of the frequency of $22.074 \mathrm{~cd}^{-1}$ also changed from year to year. It was 5.1 $\pm 0.6 \mathrm{mmag}$ during 1990-1991 (Liu Zong-Li 1993). It decreased to $2.8 \pm 0.3 \mathrm{mmag}$ during
1992-1993 (see Table 2.3). This variability in amplitudes is typical of chaotic behavior (Breger 1992).

4. As an explanation for period and amplitude variations the resonance hypothesis was discussed by some authors (Dziembowski 1980, 1982; Däppen 1985; Moskalik 1985; Takeuti 1990; Takeuti \& Zalewski 1991; Breger 1992). The key is to find the reliable interaction modes in the 0 to 3 cycles per day frequency domain to establish the link between the variability and interaction-term resonances (Breger 1992). As stated above, a frequency of $2.07 \mathrm{~cd}^{-1}$ was found in the fivefrequency solution of the 1992 data. And a frequency of $2.29 \mathrm{~cd}^{-1}$ was found in the five-frequency solution of the 1993 data. A more accurate value of $2.086 \mathrm{~cd}^{-1}$ was obtained from the combined 1992-1993 data set (see Table 2.3). It confirmed that there was a pulsation frequency really in the frequency domain from 0 to $3 \mathrm{~cd}^{-1}$ during $1992-1993$. So, the $2.086 \mathrm{~cd}^{-1}$ might be the interaction mode from which the resonances can occur and then lead to the frequency and amplitude variations of this star.

Acknowledgements. The author would like to express his thanks to National Natural Science Foundation of China for the research grants.

\section{References}

Breger M., 1990, Comm. Asteroseismology 20, Austrian Acad. Sciences, Vienna

Breger M., 1992, Comm. Asteroseismology 42, Austrian Acad. Sciences, Vienna

Däppen W., 1985, in: Buchler J.R., Perdang J.M., Spiegel E.A. (eds.) Chaos in Astrophysics. Reidel, Dordrecht, p. 273 
Dziembowski W.A., 1980, Lect. Notes Phys. 125, 22 Dziembowski W.A., 1982, Acta Astron. 32, 147

Hao J.X., 1991, Pub. Beijing Astron. Observ. 18, 35

Heynderickx D., 1990, A\&A 232, 79

Liu Z.L., 1993, A\&A 274, 220

Moskalik P., 1985, Acta Astron. 35, 229
Shi C.M., Du B.T., Jiang Z.J., Wang X.H., 1987, Acta Astrophys. Sin. 7, 230

Takeuti M., 1990, Delta Scuti Star Newslett. 2, 8, Austrian Acad. Sciences, Vienna

Takeuti M., Zalewski J., 1991, Delta Scuti Star Newslett. 4, 14, Austrian Acad. Sciences, Vienna 